\title{
Constraining D-foam via the 21-cm line
}

\author{
John Ellis, ${ }^{1,2}$ Nick E. Mavromatos, ${ }^{1}$ and Dimitri V. Nanopoulos ${ }^{3,4,5}$ \\ ${ }^{1}$ Theoretical Particle Physics and Cosmology Group, Department of Physics, King's College London, \\ Strand WC2R 2LS, London, United Kingdom \\ ${ }^{2}$ National Institute of Chemical Physics and Biophysics, Rävala 10, 10143 Tallinn, Estonia; \\ Theoretical Physics Department, CERN, CH-1211 Geneva 23, Switzerland \\ ${ }^{3}$ George P. and Cynthia W. Mitchell Institute for Fundamental Physics and Astronomy, \\ Texas A \& M University, College Station, Texas 77843, USA \\ ${ }^{4}$ Astroparticle Physics Group, Houston Advanced Research Center (HARC), \\ Mitchell Campus, Woodlands, Texas 77381, USA \\ ${ }^{5}$ Division of Natural Sciences, Academy of Athens, Athens 106 79, Greece
}

(Received 22 October 2018; published 22 January 2019)

\begin{abstract}
We have suggested earlier that D-particles, which are stringy space-time defects predicted in brane-inspired models of the Universe, might constitute a component of dark matter, and that they might contribute to the masses of singlet fermions that could provide another component. Interactions of the quantum-fluctuating D-particles with matter induce vector forces that are mediated by a massless effective U(1) gauge field, the "D-photon," which is distinct from the ordinary photon and has different properties from dark photons. We discuss the form of interactions of D-matter with conventional matter induced by D-photon exchange and calculate their strength, which depends on the density of D-particles. Observations of the hydrogen $21 \mathrm{~cm}$ line at redshifts $\gtrsim 15$ can constrain these interactions and the density of D-matter in the early Universe.
\end{abstract}

DOI: 10.1103/PhysRevD.99.015031

\section{INTRODUCTION}

The nature of dark matter (DM) is one of the biggest mysteries in cosmology and particle physics. It is commonly thought to be composed of one or more unknown species of particles, with popular candidates ranging from ultralight bosons such as axions, through sterile neutrinos and $\mathrm{TeV}$-scale to supermassive metastable particles [1]. Alternatively, the possibility that dark matter might be composed of black holes has gained in interest following the recent observations of gravitational waves emitted by mergers of black holes [2]. Another current of opinion is that dark matter might be due to some unexpected gravitational phenomenon [3]. We have been pursuing a scenario for dark matter that is complementary to these approaches, though with some aspects in common.

Our starting point is an attempt to model quantum fluctuations in space-time_- "space-time foam" [4]—using elements derived from string theory [5], in which the Universe may be modelled as a three-brane world moving in a higher-dimensional bulk space [6-11]. Generic string

Published by the American Physical Society under the terms of the Creative Commons Attribution 4.0 International license. Further distribution of this work must maintain attribution to the author(s) and the published article's title, journal citation, and DOI. models predict the appearance in this bulk space of pointlike space-time defects called D-particles [12], such as D0branes in Type IIA strings and D3-branes wrapped around appropriate three-cycles in Type IIB strings. As the threebrane world and the D-particles move in the bulk, they may encounter each other, in which case an observer on the three-brane perceives the D-particle defects as flashing on and off, giving the $3+1$-dimensional space-time a foamy structure. In such a scenario, ordinary matter and radiation are represented by open strings with their ends attached on the three-brane.

Some of the D-particles may be trapped on the threebrane, in which case they would act as dark matter $[13,14]$. However, depending on the dynamics of the bulk and the three-brane, the density of D-particles on the three-brane may evolve differently from the conventional dustlike dilution of matter density as the Universe expands. The D-particles interact with conventional matter particles via the capture and subsequent reemission of open strings, accompanied by recoil of the D-particle [15]. Such interactions with D-particles could contribute to the masses of singlet fermions that would also contribute to the dark matter density [16]. The (quantum-fluctuating) D-foam defects break Poincaré invariance, and their recoil during the interaction with propagating open-string states breaks Lorentz invariance. The nontrivial momentum transfer during the interactions of matter strings with the defects is mediated by nonlocal 
intermediate string states that do not admit a local effective action description, leading to a violation of Lorentz invariance that is subject to probes using astrophysical sources $[9,10,17,18]$. The recoil interaction of conventional matter with the D-particle defects can be described in terms of an effective U(1) gauge field - the D-photon-whose strength depends on the density of D-particles [6,7,9-11,16].

The purpose of this paper is to analyze in more detail the form of this D-particle/matter interaction, calculating its strength and discussing constraints on its strength. We recall that there are important constraints on the density of dark matter and the strength of its interactions with conventional matter at the current epoch. However, there are relatively few constraints from earlier cosmological epochs. The formation of astrophysical structures requires the presence of dark matter at redshifts $0<z \lesssim 1000$. The pattern of anisotropies in the cosmic microwave background radiation $(\mathrm{CMB})$ is sensitive to the density of dark matter at a redshift $z \sim 1000$, and there are weak constraints on dark matter/ordinary matter interactions at this and higher redshifts.

A new window on the interactions between dark matter and conventional matter has been opened up by the possibility of measuring features in the spectrum of the $21-\mathrm{cm}$ radiation emitted during transitions between the triplet and singlet states of the hydrogen atom that occurred at redshifts $z \in(15,20)$, prior to the so-called cosmic dawn. The amplitude of such a prospective signal is given by

$$
T_{21}=35 \mathrm{mK}\left(1-\frac{T_{\gamma}}{T_{s}}\right) \sqrt{\frac{1+z}{18}},
$$

where $T_{\gamma}$ is the CMB temperature and $T_{s}$ is the singlet/triplet spin temperature of the hydrogen gas, which is defined by the relative populations of the lowest-energy spin- 0 and spin- 1 states. Standard arguments within the $\Lambda$ CDM cosmological model imply the relation $T_{\gamma} \gg T_{s} \gtrsim T_{\text {gas }}$ at $z \simeq 20$, where $T_{\text {gas }}$ is the temperature of the hydrogen gas (essentially the baryon kinetic temperature, $T_{k}$ ), which is expected to be $\left.T_{\text {gas }}\right|_{z \simeq 17} \simeq 6.8 \mathrm{~K}$, leading on the basis of (1.1) to the expectation that $T_{21}>-0.2$.

Interest in this new window on dark matter has been stimulated recently [19-26] by the claim of a strong absorption feature at redshifts $z \simeq 17$ in the sky-averaged 21-cm spectrum by the EDGES Collaboration [27], which is considerably larger then the standard $\Lambda \mathrm{CDM}$ expectation. However, we do not commit ourselves to this interpretation of the EDGES data, which has not been accepted unanimously [28]. Rather, we discuss how this Type of observation can be used to constrain the D-particle model for dark matter that we introduced above.

The constraint arises from the possibility of D-photon exchange between particles of dark matter and ordinary matter, which would have effects similar to the exchange of a conventional photon or a (near-)massless dark photon
[29]. These would generate extra interactions of baryons with the dark matter fluid, beyond those predicted within the standard $\Lambda$ CDM model. Such interactions would cause the baryons to lose kinetic energy and cool down more than in the $\Lambda \mathrm{CDM}$ framework.

As a prototypical example of such a scenario, it was proposed in [19] that if the dark matter consists of more than a single dominant species, a fraction $f$ due to a millicharged component with mass $m_{\chi}$ :

$$
\left(\frac{m_{\chi}}{\mathrm{MeV}}\right) 0.0115 \% \lesssim f \lesssim 0.4 \%
$$

and an electric charge in the range $10^{-6}-10^{-4}$ of the electron charge could be consistent with the baryon cooling interpretation of the EDGES data [22,23]. This could be consistent with other existing stringent constraints, including those stemming from big bang nucleosynthesis, if $m_{\chi}$ is in a mass range much lower than the standard WIMP, $m_{\chi} \in(5-35) \mathrm{MeV}$ [20,21]. However, some degree of fine-tuning would be needed in order to mitigate the effects of several astrophysical sources of baryon heating, which make it difficult to attain the level of baryon cooling required to explain the $21 \mathrm{~cm}$ signal [21]. Interactions of baryons with (millicharged) DM particles mediated by dark photons that mix with ordinary photons [29] have also been considered in this context [24]. ${ }^{1}$

The framework we consider here is a multicomponent dark matter model, in which one of the components is a warm sterile Dirac neutrino with a mass in the range of a few tens of $\mathrm{keV}$ [32] with strong (compared to the weak standard model sector) self-interactions mediated by dark photons, whose role is played here by D-photons. This contribution to the dark matter can play a crucial role in providing the observed halo-core structure of galaxies, and contribute towards the alleviation of discrepancies between predictions based on numerical simulations based on the $\Lambda$ CDM model and observations at galactic scales (the so-called "smallscale" cosmology crisis [33]).

We discuss here the contribution to elastic scattering with such sterile neutrinos mediated by the exchange of the effective D-photon U(1) gauge field induced by the recoil of D-particles, and calculate its magnitude. We discuss how this interaction could contribute to baryon cooling, and show how any upper limit on such an effect could be used to constrain the properties of D-foam.

The structure of the paper is as follows. In Sec. II we review the main features of the stringy D-foam model, which offers a microscopic multispecies dark matter model

\footnotetext{
${ }^{1}$ The stringent constraints on millicharged dark matter are avoided in models for baryon cooling via interactions with axion condensates $[25,26]$, either of quantum chromodynamics (QCD) origin [30], or string-inspired [31], but these are subject to other astrophysical constraints.
} 
framework within brane/string theory. In the following Sec. III we discuss the strength of the D-photon-mediated interactions between baryonic matter and singlet sterile Dirac neutrinos, and how these interactions could contribute to baryon cooling. Our conclusions and outlook are summarized in Sec. IV. In an Appendix we discuss some complimentary aspects of D-matter interactions with standard model particles, namely universal lensing properties of D-foam originating from the backreaction on space-time of the recoiling D-particles during their interaction with generic matter.

\section{A MODEL FOR D-FOAM INTERACTIONS WITH SINGLET FERMIONS}

We advocate a (toy) model of space-time foam [4], motivated by string theory [6-11], in which (effectively pointlike) compactified braney stringy defects [5], termed D-particles, provide foamy space-time structures as illustrated in Fig. 1. Our Universe is described as a three-brane world moving in a higher-dimensional bulk space that is punctured by D-particle defects. As the brane world and the D-particles move in the bulk, they may encounter each other, and an observer on the D3-brane would see the

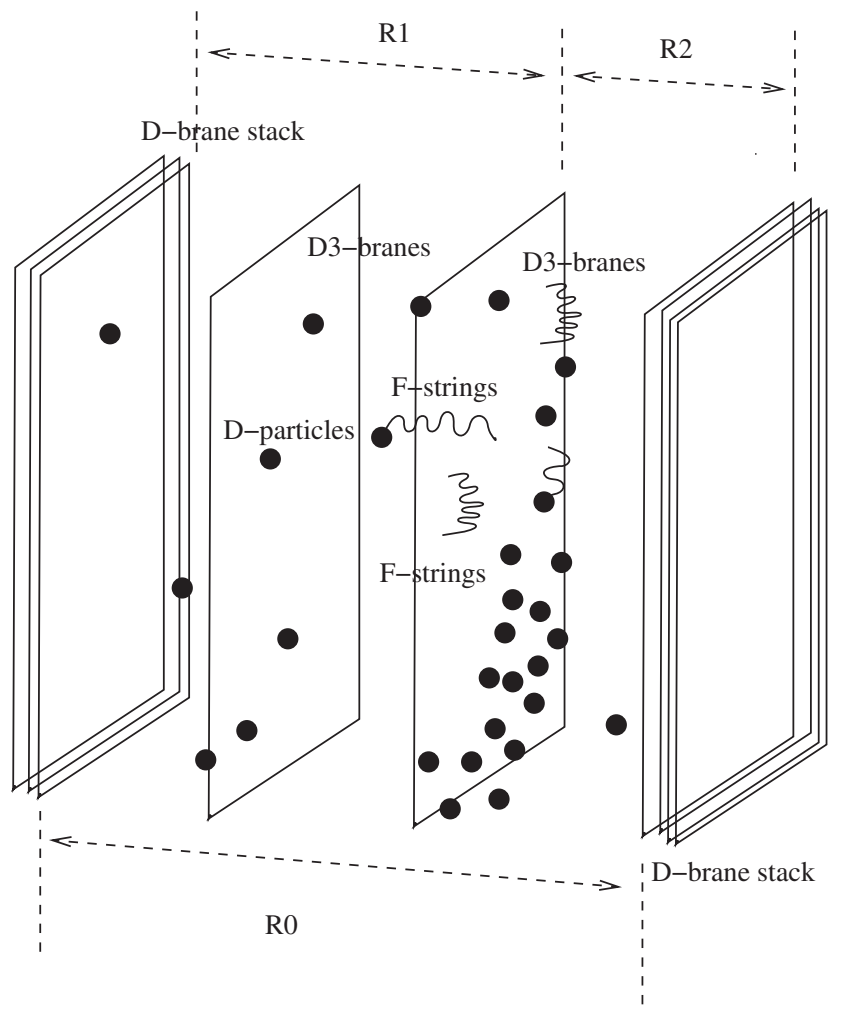

FIG. 1. Schematic representation of a string/brane-theory-inspired prototype of a D-particle model of space-time foam [6] proposed in [7]. D-particles are represented by dots, and conventional particles are excitations on D3-branes that may be connected to D-particles.
D-particle defects as flashing on and off, thus giving the space-time a foamy nature [4].

In addition, some of the D-particles may be trapped on our three-brane, contributing to the density of cold dark matter. However, the density of these defects is not necessarily uniform, and the effective density of D-particles on our three-brane world varies with the cosmic redshift in a model-dependent way that is linked to the density profiles of the D-particles in the bulk space. For this reason, the D-particle density may not evolve with redshift in the same way as conventional dustlike dark matter.

Depending on the string theory considered, the D-particles may either be point-like D0-branes (as in Type IIA strings) [6,7,9] or D3-branes wrapped around appropriate three-cycles (as in Type IIB strings [10]). In such scenarios, ordinary matter and radiation are represented by open strings with their ends attached on the D3-brane. The D-particles interact with standard model matter particles in a topologically nontrivial manner, via the capture and reemission of the open strings, accompanied by recoil of the D-particle, as illustrated in Fig. 1. The D-foam defects break Poincaré invariance, and these interactionswhich are mediated by a nonlocal intermediate $U(1)$ vector field-cause Lorentz invariance to be broken in the propagation of open string states.

In our approach $[6,9,10]$ the D-foam defects are to be thought of as providing an environment or background medium, analogous to the lattice of ions in a solid-state system, and the $\mathrm{U}(1)$ vector field mediating the interaction with matter particles is analogous to a phonon excitation [16]. Its vector nature is due to its role as a Goldstone boson associated with the spontaneous breaking of Lorentz symmetry due to the recoiling D-particle defect. We recall that the strength of the D-foam-matter interactions is not universal for different particle species. In particular, the interactions are nontrivial only for photons and any other neutral particles that have no nontrivial internal quantum numbers [8,9], for which there is no obstacle to absorption and reemission by a D-particle. ${ }^{2}$

It is well known that, in string theory, electrodynamics is described by a Born-Infeld extension of the minimal Maxwell Lagrangian of conventional QED with photon field $A_{\mu}$ and field strength $F_{\mu \nu}$. Moreover, it was argued in $[13,14,16]$ that the effective low-energy theory describing the interaction of the D-foam with matter strings is also represented by Born-Infeld-Type dynamics for the D-photon field $\mathcal{A}_{\mu}$. Their combination, including the D-photon interaction with a fermion current, can be written in a manifestly dual form [35] as:

\footnotetext{
${ }^{2} \mathrm{~A}$ form of D-brane dark matter has also been proposed independently in [34], but from a rather different perspective. In particular, that model did not have nonuniversal couplings to different matter species.
} 


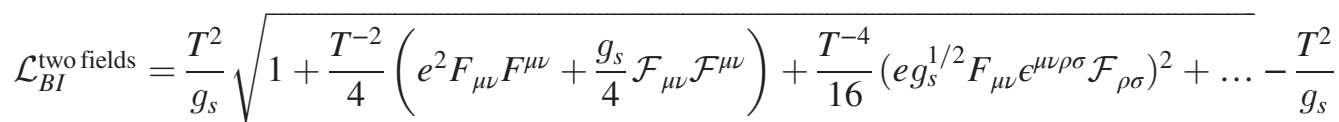

$$
\begin{aligned}
& +\sum_{I=b, N} \int d^{4} x \tilde{g}_{V, I} \mathcal{A}_{\mu} \bar{\psi}_{I} \gamma^{\mu} \psi_{I}+\sum_{I=b, N} \int d^{4} x \bar{\psi}_{I} i \partial / \psi_{I}+\ldots
\end{aligned}
$$

in the notation of [16], where we refer the reader for details. Here the quantity $\mathcal{F}_{\mu \nu}=\partial_{\mu} \mathcal{A}_{\nu}-\partial_{\nu} \mathcal{A}_{\mu}$ denotes the field strength tensor of the vector field $\mathcal{A}_{\mu}, T \equiv 1 /\left(2 \pi \alpha^{\prime}\right)$ where $\alpha^{\prime}=M_{s}^{-2}$ is the string tension with $M_{s}$ the string mass scale, which is in general lower than the four-dimensional Planck mass [5], $g_{s}$ is the string coupling, ${ }^{3}$ the $\ldots$ in the upper line denote $B$-field terms, and the $\ldots$ in the lower line denote other fields as well as the higher-derivative terms that appear in string effective actions. The fermion fields $\psi_{I}, I=b, N$ may represent either a baryon $(I=b)$ or a singlet fermion such as a sterile neutrino $(I=N)$. The reader should remember that in open string theory [5], the electron charge $e$ is expressed in terms of the string coupling as:

$$
g_{s}=e^{2},
$$

which stems from the requirement that the electromagnetic part of the low-energy Born-Infeld terms in the targetspace-time Lagrangian that describes the propagation of open strings in an electromagnetic background should yield the canonically-normalized Maxwell action to lowest order in derivatives. ${ }^{4}$

As explained in $[11,16]$, in addition to the recoil vector contributions in (2.1), there are also contributions arising from the antisymmetric (spin-one) tensor field $\hat{B}_{\mu \nu}$ in the massless string multiplet. In our approach (through summation of world-sheet genera to encapsulate the quantum string loop effects properly), this has been argued to be a derivative operator whose components are given by

$$
\begin{aligned}
\hat{B}_{i 0} & =\hat{u}_{i}=-i g_{s} \frac{r_{i}}{M_{s}} \frac{\partial}{\partial X^{i}} \\
& \equiv-i g_{s} \frac{r_{i}}{M_{s}} \nabla_{i}(\text { no sum over } i=1,2,3), \\
\hat{B}_{i j} & =\epsilon_{i j k} \hat{u}^{k},
\end{aligned}
$$

\footnotetext{
${ }^{3}$ It should be understood that in the presence of a nontrivial, space-time coordinate-dependent dilaton field, $\Phi(x)$, the string coupling $g_{s}$ in (2.1) would be replaced by $e^{\Phi(x)} g_{s}$.

${ }^{4}$ We note that any mixing between the D-photon and the ordinary photon in (2.1) occurs at fourth order in the derivative expansion. Thus, there is no kinetic mixing at second-derivative order, as in standard dark photon models. Truncating the derivative expansion of the Born-Infeld square root to second order is sufficient for our purposes, in which case D-photon/ photon mixing can be neglected.
}

where $r_{i}$ denotes the fraction of momentum transferred during each collision of a matter string with a D-particle defect. Making a derivative expansion of the effective action, and averaging stochastically over the population of D-particles, with $\left\langle r_{i}\right\rangle=0$, and $\left\langle r_{i} r_{j}\right\rangle=\sigma^{2} \delta_{i j}$, these terms yield quartic (and higher-order) Lorentz-violating terms in the Lagrangian of the form

$$
\mathcal{L}_{\text {B-field }} \ni \frac{1}{16} \mathcal{F}_{\mu \nu} \frac{g_{s}^{2} \tilde{\sigma}^{2}}{M_{s}^{2}} \Delta \mathcal{F}^{\mu \nu}
$$

where $\Delta$ is the 3 -space Laplacian, $\Delta \equiv \nabla_{i} \nabla^{i}=\vec{\nabla} \cdot \vec{\nabla}$. The quantity $\tilde{\sigma}^{2} \propto \sigma^{2}$, where the numerical proportionality factor is associated with quantum ordering ambiguities [11], and can be absorbed in the definition of the stochastic variance. This is treated here as a phenomenological parameter characterizing the foam, which depends in general on its density. It was argued in [16] that these terms would generate dynamical masses for the singlet fermions, as discussed briefly below.

The baryons $\psi_{b}$ are Dirac fermions. On the other hand, depending on the underlying microscopic model, the singlet fermions $\psi_{N}$ could be either Dirac or Majorana. In the latter case, the Majorana fermion field $\psi_{N}^{M}$ could have only axial-vector couplings with the $\mathcal{A}$ vector field, as follows from charge conjugation of the Majorana field. Therefore, the effect of the dark matter particles on baryon cooling through D-photon exchanges would be negligible in the Majorana case, ${ }^{5}$ unless standard model fermions $(f)$ also have axial couplings $\tilde{g}_{A, I}, I=f, N$ with the D-photon $\mathcal{A}_{\mu}$, mimicking $Z$-portal models for DM [36]:

$\mathcal{L}_{\text {fermion-D-foam-axial }} \ni \sum_{I=b, N} \int d^{4} x \tilde{g}_{A, I} \mathcal{A}_{\mu} \bar{\psi}_{I} \gamma^{\mu} \gamma^{5} \psi_{I}$.

The analysis of elastic scattering involving axial current couplings to DM and nucleons in the Majorana case is entirely analogous to the case of Dirac DM, which we consider below for concreteness.

Thus, in the case of either Dirac or Majorana singlet DM fermions, vector or axial vector interactions between the

\footnotetext{
${ }^{5}$ Moreover, the cooling of baryons through their direct interaction with the D-foam would in general be too weak to be of phenomenological interest, though condensates of D-particles could enhance the effect, as discussed briefly at the end of Sec. III.
} 
$\mathcal{A}$-field and the fermions are allowed, as in (2.1), (2.5), and this may lead to an interesting mechanism for baryon cooling through the elastic scattering of baryons with singlet fermions, via tree-level $\mathcal{A}$ exchange, mimicking, straightforwardly, the millicharged DM case. But such a similarity is only formal, given that the $D$-photon coupling is not an electromagnetic one, as already explained.

In what follows we shall restrict for concreteness our discussion to the Dirac DM fermion case. We note at this point, that, as follows from our formalism (2.1), Dirac antifermions couple to the $\mathcal{A}$-field with couplings $\tilde{g}_{V(A), I}$ of opposite sign. This stems from the CPT properties of the Dirac equation in the low-energy effective action (2.1), and is consistent with the gauge nature of the recoil excitations of the D-particles, which are represented by open strings stretched between the D-particle defect and the brane in the T-dual formalism [15]. For Majorana particles, on the other hand, the axial coupling (2.5) guarantees that particles and antiparticles have identical couplings. We note that, since the D-particle recoil interaction is mediated by a spin-one open string excitation, in general it need not be the same for particles and antiparticles. This is indeed the case for a Dirac DM fermion, which may have either vector and/or axial-vector recoil interactions [10].

The couplings ${ }^{6} \tilde{g}_{V, I}, I=b, N$ are in general of different strengths, as discussed above, in particular $\tilde{g}_{V b}=0$ in Type IA models. The factor $g_{s}^{1 / 2}$ pulled out explicitly in front of $\mathcal{F}$-terms in the argument of the square root in (2.1) ensures the canonical normalization of the Maxwell-like kinetic term of the $\mathcal{A}_{\mu}$ gauge field, when the square root is expanded in powers of $T^{-1}$. Since we are interested here only in low-energy dynamics, we neglect the higher-order terms in $\mathcal{F}_{\mu \nu}$ in (2.1), and restrict our attention to the leading-order Maxwell term.

To summarize, the relevant low-energy part of the effective target-space action on our three-brane world, where the quantum fluctuating D-particle meets the open-string singlet fermion state, is described by the Lagrangian (2.1) expanded in powers of derivatives:

$$
\begin{aligned}
S= & \int d^{4} x\left[\frac{1}{4} \mathcal{F}_{\mu \nu}\left(1+\frac{1}{4} \frac{g_{s}^{2} \tilde{\sigma}^{2}}{M_{s}^{2}} \Delta\right) \mathcal{F}^{\mu \nu}\right] \\
& +\sum_{I=b, N} \int d^{4} x \tilde{g}_{V, I} \mathcal{A}_{\mu} \bar{\psi}_{I} \gamma^{\mu} \psi_{I} \\
& +\sum_{I=b, N} \int d^{4} x \bar{\psi}_{I} i \partial / \psi_{I}+\ldots,
\end{aligned}
$$

where the ... represent terms of higher order in derivatives as well as elecromagnetic and other fields in the string

\footnotetext{
${ }^{6}$ In what follows we restrict ourselves for concreteness to vector couplings, however entirely analogous considerations characterize the axial D-photon coupling to fermions.
}

effective action. The Lorentz-violating terms in (2.6) that are quartic in derivatives are relevant for the generation of nonperturbative masses for singlet fermions $\psi_{I}$, as discussed in $[11,16]$ :

$$
\begin{aligned}
& m_{d y n}^{I} \simeq M \exp \left(-\frac{2 \pi}{3 \alpha_{V, I}}\right), \quad I=b, N, \\
& \alpha_{V, I}=\frac{\tilde{g}_{V, I}^{2}}{4 \pi}, \quad M=\frac{M_{s}}{g_{s} \sqrt{\tilde{\sigma}^{2}}},
\end{aligned}
$$

but do not play any role in baryon cooling,

The magnitude of the dimensionless coupling $\tilde{g}_{V}$ depends on the string model. As discussed in [16], in the context of the Type IIB model of D-foam [10], in which our world is viewed as a D7 brane with four dimensions compactified, and the "D-particles" of the foam are represented by compactified D3 branes wrapped around three cycles, we find for the coupling of the D-foam to singlet fermions

$$
\tilde{g}_{V, N} \propto g_{s}^{1 / 2} \sqrt{n_{D}^{(3)} R^{\prime-1}} \mathcal{F}\left(s, t, \alpha^{\prime}\right) M_{s}^{-2},
$$

where $n_{D}^{(3)}$ is the density of D-particles, and $R^{\prime}$ is the radius of the fourth space dimension of the D7 brane transverse to the D3 brane. A phenomenological estimate is $R^{\prime} \sim 338 M_{s}^{-1}$ in the model of [10], but different values are possible in other models. The quantities $s$ and $t$ in (2.8) are Mandelstam variables and $\mathcal{F}\left(s, t, \alpha^{\prime}\right)$ is a momentum-dependent form factor associated with string amplitudes describing the scattering of such singlet fermionic excitations off D-particles in the model, including string-loop corrections that are suppressed by powers of $g_{s}$ that modify the coupling $\tilde{g}_{V}$. This form factor is difficult to compute exactly, given that the target-space action of D-branes is not fully known. However, for the slowly-moving excitations with momenta that are small compared to the string mass scale $M_{s}$ that are of relevance here, a field-theoretical approximation is fully adequate, and the form factor is well approximated by unity.

In the Type IIB string-foam model of [10], electricallycharged baryons (or electrons) interact with the recoiling D-foam with a coupling that is very suppressed to (2.8):

$$
\begin{aligned}
\tilde{g}_{V, b} & =\left(1.55 M_{s}^{-1}\right)^{2} \sqrt{n_{D}^{(3)} R^{\prime-1}} \tilde{g}_{V, N} \\
& \propto g_{s}^{1 / 2}\left(n_{D}^{(3)} R^{\prime-1}\right)\left(1.55 M_{s}^{-1}\right)^{2} \ll \tilde{g}_{V, N} .
\end{aligned}
$$

We note in passing that in [16] we discussed a geometric mechanism that enhances the dynamical fermion mass (2.7) via a suitable embedding of the model in a higherdimensional set-up involving brane worlds [37] in a Randall-Sundrum (RS) warped bulk geometry [38]. By selecting appropriately the parameters of such an embedding one can generate a mass for the sterile neutrinos that is 
of phenomenological interest. ${ }^{7}$ Thus, it was argued in $[11,16]$ that another observable implication of D-foam could be the dynamical generation of small nonperturbative masses for singlet neutral fermions in Type IA D-foam models, where the foam is transparent to charged fermions, and for both singlet neutral and charged fermions in Type IIB D-foam models, though the charged and singlet fermions couple with different strengths to the D-foam.

We shall not discuss dark energy here, assuming that it is small at the epoch of interest. In the presence of a nontrivial, space-time-coordinate dependent dilaton field, $\Phi(x)$, the string coupling $g_{s}$ in (2.1) would be replaced by $e^{\Phi(x)} g_{s}$. However, we assume that the value of the dilaton field $\Phi$ is constant during this era, and in general at later epochs of the Universe, and normalize it to zero.

\section{BARYON COOLING IN D-FOAM}

In this section we consider the possible contribution of D-foam to baryon cooling, focusing on the Type IIB model of [10]. The effect in Type I string D-foam is strongly suppressed because there is no direct interaction of the D-photon with the baryon (or charged matter more generally). In this model, the only way to generate an interaction between dark matter and ordinary matter would be through the kinetic mixing of the D-photon with the ordinary photon. However, in our Born-Infeld model (2.1) this happens only at four-derivative order, and involves two D-photons and two ordinary photons. In the Type IIB model, a D-photon mediator can directly couple with a baryon, yielding a $v^{-4}$ Rutherford-like behavior of the dark matter/baryon scattering cross section. This can be significant if the dark matter has a warm component of sterile neutrinos, with mass a few tens of $\mathrm{keV}$, as in the model of [32]. In this case, the reduced mass $\mu_{N, b}$ will be of order of the sterile neutrino mass, which is much smaller than the baryon mass, so the cross-section can be much larger than that for other possible dark matter species with masses $m_{\chi} \gg 1 \mathrm{GeV}$ that might interact with the baryons, thereby providing the leading cooling mechanism for baryons, beyond the standard cosmological model, leading to strong potential constraints on the D-foam parameters as we discuss below. ${ }^{8}$

\footnotetext{
${ }^{7}$ However, standard model fermions acquire their masses from the conventional Higgs mechanism.

${ }^{8}$ As was already stressed in the previous section, the D-particles, with masses $M_{s} / g_{s} \sim M_{s} /(4 \pi \alpha) \gg \mathrm{TeV}$ are assumed not to propagate like ordinary excitations. Instead, in a low-energy limit, their interaction with ordinary particle excitations is expressed via the effective recoil vector field $\mathcal{A}_{\mu}$ that mediates dark matter/baryon interactions. However, this D-foam background may exhibit nontrivial optical properties (refractive indices), which have been studied elsewhere $[9,10,18]$ and provide complementary phenomenological constraints on the D-foam model.
}

Using the Lagrangian (2.6) and ignoring higher-derivative Lorentz-violating terms, one can readily calculate the total cross section for elastic scattering of sterile neutrinos on baryons, which is mediated by tree-level $\mathcal{A}_{\mu}$ exchange, and has the same form as the QED Rutherford cross section. It may be written in the form $[20,23]$

$$
\sigma_{t}=\int d(\cos \theta)(1-\cos \theta) \frac{d \sigma(\theta)}{d(\cos \theta)}
$$

where $\theta$ is the scattering angle, and

$$
\frac{d \sigma(\theta)}{d(\cos \theta)}=-\frac{1}{2 \pi} \int_{0}^{2 \pi} \frac{d \sigma}{d \Omega} d \phi
$$

where $d \sigma / d \Omega$ is the differential cross section in the solid angle $\Omega$, which is inversely proportional to the fourth power of $v$, exactly as in the case of millicharged dark matter/-baryon scattering [21], but with the electric charge of the baryon replaced by the coupling $\tilde{g}_{V, b}(2.9)$, and the charge $\epsilon e$ of the millicharged dark matter particle replaced by $\tilde{g}_{V, N} \gg \tilde{g}_{V, b}$ :

$$
\begin{aligned}
\sigma_{t} & \simeq \frac{2 \pi \alpha_{V, N}^{2}\left(n_{D}^{(3)} R^{\prime-1}\right)\left(1.55 M_{s}^{-1}\right)^{4}}{\mu_{N, b}^{2} v^{4}} \xi \\
& =\frac{2 \pi \alpha^{2}\left(n_{D}^{(3)} R^{\prime-1}\right)^{2}\left(1.55 M_{s}^{-2}\right)^{4}}{\mu_{N, b}^{2} v^{4}} \xi,
\end{aligned}
$$

where $\mu_{N, b}=\frac{m_{N} m_{b}}{m_{N}+m_{b}}$ is the reduced mass of the sterile neutrino/baryon system, $m_{N}\left(m_{b}\right)$ being the mass of the sterile neutrino (baryon). The quantity $\alpha_{V, N}$ was defined in (2.7), and $\alpha=\frac{e^{2}}{4 \pi}$ is the fine structure constant of electromagnetism. In expressing the result in terms of $\alpha$, we took into account (2.2). The quantity $\xi$ in (3.3) corresponds to the logarithmic Debye regulator function for the forward divergence of the momentum transfer integral in QED [22], which we discuss below.

Comparing the cross section (3.3) with the corresponding one for millicharged dark matter/baryon interactions [21], the role of the millicharge parameter $\epsilon=e_{\chi} / e$ is played here by the quantity

$$
\varepsilon \equiv\left(n_{D}^{(3)} R^{\prime-1}\right)^{1 / 2}\left(1.55 M_{s}^{-1}\right),
$$

in terms of which the cross section (3.3) acquires a form similar to that for millicharged dark matter/baryon scattering [21]:

$$
\sigma_{t} \simeq \frac{2 \pi \alpha^{2} \varepsilon^{4}}{\mu_{N, b}^{2} v^{4}} \xi
$$

However, in contrast to the millicharged case, here both the baryon and the singlet fermion carry "charge" $\varepsilon e$ for the 
D-photon $\mathrm{U}(1)$ gauge group. The quantity $\varepsilon$ depends on the density of the D-foam $n_{D}(z)$, which is in general redshiftdependent, as mentioned earlier, and the compactification parameters of the underlying Type IIB string theory model [10]. Constraints on baryon cooling at redshifts $z \simeq 15$ to 20 [27] can be used to bound these model parameters.

Before discussing this, we first discuss the Debye function $\xi$ appearing in (3.3). As in the QED case, the differential cross section

$$
\frac{d \sigma}{d(\cos \theta)}=\frac{2 \pi \varepsilon^{4} \alpha^{2}}{\mu_{N, b}^{2} v^{4}}\left(\frac{1}{1-\cos \theta}\right)^{2}
$$

diverges when the forward scattering angle $\theta \rightarrow 0$. In the QED case this regularized [22] by the Debye screening due to free electrons and protons in the plasma at redshifts $z \lesssim 20$, with a density $n_{e}=n_{p}=\Omega_{b} \rho_{c} a_{R}^{-3} / m_{p}$, where $\Omega_{b}$ is the baryon density normalized with respect to the critical density $\rho_{c}, a_{R}$ is the Universe scale factor at recombination, and $m_{p}$ is the proton mass. In millicharged dark matter scenarios, it is assumed that the dark matter is tightly coupled to the photon-baryon plasma by Coulomb scattering due to photon exchange, so that the dark matter density fluctuations are damped.

In our D-foam case, although the dark matter particle is electrically neutral, it has a D-foam U(1) "charge" $\varepsilon e$, while electrons and baryons carry both the electric $e$ and the D-foam U(1) charge $\varepsilon^{2} e$, see (2.9), (3.4). Since the coupling of the dark matter to baryons provided by this charge is dependent on the density of foam, constraints on the latter can be derived by assuming that it is not strong enough to affect the relative velocity difference between DM and baryons prior to recombination as in the millicharged case [20], or damp significantly the baryon acoustic oscillations [26], which would lead to inconsistencies with observations.

Under this assumption, one can adapt the regularization procedure of [22] to this case, The divergence for forward scattering encountered in (3.6) is removed by replacing the zero of the scattering angle $\theta \rightarrow 0$ by a minimum nonzero angle $\theta_{\min } \ll 1$, which is related to a maximum impact parameter $b_{\max }$ for dark matter/baryon scattering [22]:

$$
b_{\max }=\frac{\varepsilon^{3} \alpha}{\left\langle\mu_{N, b} v^{2}\right\rangle} \cot \left(\frac{\theta_{\min }}{2}\right) \stackrel{\theta_{\min } \ll 1}{\simeq} \frac{\varepsilon^{3} \alpha}{3 T} \frac{2}{\theta_{\min }},
$$

where we have used $\left\langle\mu_{N, b} v^{2}\right\rangle=3 T$, as appropriate for thermalized particles. In the case of standard electromagnetic scattering, one would equate this maximum impact parameter to the corresponding Debye length $\lambda_{D}$ of the baryon plasma, which indicates the maximum range of the electrostatic effect of a charged carrier. In our case, the analogue of the Debye length for the effective U(1) interaction is not known: the full Born-Infeld Lagrangian (2.1) replaces the Maxwell part of the action (2.6), together with higher-order covariant-derivative terms acting on fermions, whose full form depends on the specific microscopic string model considered and cannot be expressed in closed form. Nonetheless, one can make the simplifying assumption that the $\mathrm{U}(1)$ interactions of the dark matter with the baryons would mimic the standard electromagnetic expression for the Debye length for a baryon population ("plasma") in thermal equilibrium with the D-foam at a temperature $T .{ }^{9}$ This follows from the fact that a Debye length scale

$$
\lambda_{D}=\left(\frac{\epsilon T}{\sum_{j=1}^{N} n_{j} q_{j}^{2}}\right)^{1 / 2},
$$

arises in thermodynamic descriptions of large systems consisting of $N$ charged species with mobile charges $q_{j}$ and (number) densities $n_{j}$ in a medium with static permittivity $\epsilon$, following the corresponding Poisson equation. The similarity of the low-energy theory of the effective D-foam interactions with standard electromagnetism implies that in our D-foam case, where the baryons have $\mathrm{U}(1)$ charge $\varepsilon^{2} e$ [(2.9), (3.4)], and density $n_{p}(z)$ at redshift $z$, the corresponding Debye screening length for D-foam effects in a baryon plasma at temperature $T_{b}(z)$, would be

$$
\lambda_{D}^{\text {D-foam }}=\left(\frac{\epsilon T_{b}(z)}{4 \pi \varepsilon^{4} n_{b}(z) \alpha}\right)^{1 / 2} .
$$

Identifying $b_{\max }=\lambda_{D}^{\text {D-foam }}$, and setting the $\mathrm{D}$-foam vacuum permittivity $\epsilon=1$, we obtain from (3.7) and (3.9):

$$
\theta_{\min }=\frac{2 \varepsilon^{3} \alpha}{3 T_{b} \lambda_{D}^{\text {D-foam }}}=\frac{2 \varepsilon^{5} \alpha^{3 / 2}\left(4 \pi n_{b}\right)^{1 / 2}}{3 T_{b}^{3 / 2}} .
$$

Performing the regularized integration over $\theta$ in (3.1), using (3.6) and (3.10), we find the following expression for the logarithmic Debye regulator function $\xi$

$\xi=\ln \left(\frac{4}{\theta_{\min }^{2}}\right)=\ln \left(\frac{9 T_{b}^{3}}{4 \pi \varepsilon^{10} \alpha^{3} n_{b}}\right)=\ln \left(\frac{9 T_{b}^{3}}{4 \pi \alpha^{3} n_{b}}\right)-10 \ln \varepsilon$

in our case.

We note some crucial differences from the millicharged case. In the latter, the interaction between DM and baryons occurs through the conventional photon of electromagnetism, and this is why only highly-ionizing matter is relevant in that case. This implies that only the free-electron plasma fraction $x_{e} n_{H}$, where $x_{e}=n_{e} / n_{H}$, of the total density of the hydrogen gas $n_{H}$ interacts with DM, and hence in such a

\footnotetext{
${ }^{9}$ This is a rather drastic assumption, but is motivated by the fact that thermalized D-matter reproduces the correct large-scale structure of the Universe, if the D-matter constitutes a dominant dark matter component at large scales [14].
} 
case one should replace $n_{b}$ in (3.11) by $x_{e} n_{H}$. This fraction becomes very small during the cosmic dawn era: $x_{e}^{\text {dawn }} \sim$ $2 \times 10^{-4}$ [21], which is why in the millicharged DM case one would need a cross section that exceeds the CMB limits [39] by several orders of magnitude in order to reproduce the EDGES signal, assuming that millicharged particles provide all the DM. Otherwise, if the millicharged DM constitutes only a fraction $f_{\chi}<0.4 \%$ of the DM, there is a small window in the parameter space of millicharged DM that reproduces the reported EDGES signal while being consistent with the CMB constraint (1.2) [21].

Moreover, since protons, electrons and singlet fermions are all "charged" under the recoil $\mathrm{U}(1)_{D}$ gauge group in our Type IIB D-foam model, unlike electromagnetism, there is a direct coupling of the DM singlet fermions to electrically-neutral Hydrogen atoms Since the D-photon is massless, the cross section for its exchange $\propto v^{-4}$, where $v$ is the relative velocity between the singlet fermions and the hydrogen atoms, and there is a suppression factor $\alpha^{2}\left(n_{D}^{(3)} R_{10}^{\prime-1}\right)^{2}\left(1.55 M_{s}^{-2}\right)^{4}$ that depends on the density of D-foam. ${ }^{10}$

This coupling of singlet fermions to neutral hydrogen matter implies that most of the CMB constraints that are so important in the millicharged case are avoided, as discussed in [21] (see Fig. 6 and the related discussion in that work), since the population of Hydrogen gas is much greater than that of the ionized matter that is relevant in the millicharged DM case. The D-photon interactions with individual SM particles could therefore be much weaker than the interaction of the conventional photon with millicharged particles and still provide significant baryon cooling without contradicting the CMB constraints.

The quantity $\xi$ may be estimated by assuming that the baryon number density $n_{b}(z)$ during the relevant epoch $z=\mathcal{O}(15)$ is associated mainly with hydrogen (protons), and may be evaluated using the Planck measurements [39] of the current era baryon density $\Omega_{b}$, using the standard $(1+z)^{3}$ redshift scaling of cosmic matter: $n_{b}(z)=$ $\Omega_{b} \rho_{c}(1+z)^{3} / m_{p}$, where $m_{p} \simeq 1 \mathrm{GeV}$, and $\Omega_{b} \simeq 0.023 h^{-2}$, $h=0,71$, and $\rho_{c}=8.099 h^{2} \times 10^{-47} \mathrm{GeV}^{4}$. Taking a fiducial temperature for baryons $T_{b} \simeq 10 \mathrm{~K} \simeq 9 \times 10^{-13} \mathrm{GeV}$ at $z=20$ [20,21], (3.11) yields

$$
\begin{aligned}
\xi & =\ln \left(9 T_{b}^{3}\left(4 \pi n_{b} \alpha^{3}\right)^{-1}\right)-10 \ln \varepsilon \\
& =\ln \left(7.8 \times 10^{13}\right) \simeq 32-10 \ln \varepsilon
\end{aligned}
$$

for the Type IIB D-foam model. Assuming that the D-foam is in thermal equilibrium at the relevant epoch, the evolution equation for the relative velocity between baryon and DM and the cooling temperature depend on the cross

\footnotetext{
${ }^{10} \mathrm{We}$ recall that the size of the hydrogen atom is significantly larger than the Compton wavelength of a D-particle, whose mass $M_{s} / g_{s}$ is in the multi-TeV range.
}

section, as follows from the analysis in $[21,23]$. Constraints from CMB and Planck data can be taken into account by following the millicharged DM analysis. Although from this point of view the mass and the density fraction of the sterile neutrino DM are in principle adjustable parameters, we take into account the constraints derived in [32], and require masses of a few tens of $\mathrm{keV}$ for the sterile neutrinos, which may thus play an important role in galactic structure.

We now examine the bounds from Planck CMB measurements [39] on the D-photon-mediated cross section, thereby constraining the D-foam parameters. Parametrizing a generic DM-baryon cross section as $\sigma_{t}=\sigma_{0} v^{-4}$, it was demonstrated in [21] that, for DM masses less than a few $\mathrm{MeV}$, the Planck CMB constraints at redshifts $z=17$ can be satisfied for

$$
\sigma_{0} \lesssim 1.7 \times 10^{-41} \mathrm{~cm}^{2} \quad \text { at } 95 \% \text { C.L. }
$$

We then find, using (3.5), that

$$
\xi^{1 / 2} \varepsilon \lesssim 10^{-10}, \quad\left(m_{N} \ll m_{b} \sim 1 \mathrm{GeV}\right) .
$$

In the model of Type-IIB D-foam discussed in [10], the value

$$
n_{D}^{(3)} \simeq 10^{-3} M_{s}^{-3}, \quad R^{\prime} \simeq 338 M_{s}^{-1}
$$

was used for the density of D-particles, which is consistent with constraints on a possible foam-induced refractive index for photons at late redshifts $z \lesssim 1$, as well as the strong constraints on electrons coming from observations of synchrotron radiation from the Crab Nebula [18]. In this case, we obtain from (3.4)

$$
\varepsilon \simeq 7 \times 10^{-6} .
$$

Using (3.12), this implies

$$
\xi \simeq 151
$$

Comparing with (3.14), we see that the Planck CMB constraints [39] on the cross section are not satisfied for sterile neutrino DM, as the corresponding cross section exceeds the CMB limits by several orders of magnitude. One would need to postulate much more dilute foam densities at redshifts $z=\mathcal{O}(20)$, which is possible because the D-foam density is free and redshift-dependent in general, as discussed in [6].

The constraint (3.17) could be evaded if D-particles form condensates on the brane Universe, due to their stringy selfinteractions, by analogy with axion condensate formation in field [30] or string theory [31]. As discussed in [13], the fact that D-particles live in the higher-dimensional bulk implies that the bound states between D-particles and the brane world do not overclose the universe, as there are 
contributions to the brane world vacuum energy from the bulk D-particles of mixed signs, depending on their distance from the brane world [7]. As mentioned previously, coherent oscillations of a condensate of D-particles would lead to the appearance of a vector $\mathcal{A}_{\mu}$ excitation that mediates interactions between baryons and DM particles propagating in the $\mathrm{D}$-foam. The formation of a D-particle condensate would imply a coherence length that grows linearly with the scale factor of the expanding brane universe, strengthening D-foam-mediated effects in induced DM-baryon interactions. At the field-theory level, this would lead to an induced "superconductivity" phenomenon in the interactions of the recoil-induced D-photon. The "magnetic field" of the $\mathrm{U}_{D}(1)$ recoil gauge theory would be "expelled" from the region occupied by the D-particle condensate à la Meissner, which would imply a low "magnetic" permeability but high "vacuum permittivity" $\epsilon$ (i.e., a high "dielectric constant") for the D-foam "vacuum" (since their product is always $1 / c^{2}$ ). In such a case the Debye screening length (3.9) could be significantly larger than calculated above, leading to larger cross sections [see (3.5), (3.10) and (3.11)]. We leave the investigation of such models to future work.

\section{CONCLUSIONS AND OUTLOOK}

Triggered by the advent of early-Universe $21-\mathrm{cm}$ astrophysics, as heralded by the first report from the EDGES Collaboration [27], we have discussed in this paper the possible interactions between baryons and singlet DM fermions such as sterile neutrinos that could be induced by D-foam in Type-IIB string theory. As discussed in [10], the recoil of particles scattering off D-matter is mediated by an effective photonlike excitation, the D-photon. This leads to a cross section with Rutherford-like behavior, which depends on the inverse fourth power of their relative velocity, implying a significant enhancement of such interactions for slowly-moving baryons that would be most relevant during the cosmic dawn era. We have estimated in this paper the normalization of corresponding induced cross section between baryons and DM, and discussed the constraints imposed by the CMB and the possible relevance for baryon cooling at redshifts $z \sim 10$ to 20 .

The velocity dependence of D-photon exchange is the same as that of photon exchange in the millicharged DM scenario, though here the exchanged particles are not electromagnetic photons, but Goldstone bosons due to Lorentz violation associated with quantum-gravitational fluctuations of the stringy space-time foam. The corresponding coupling depends on the density of foam, which is an adjustable phenomenological parameter, together with the mass of the singlet fermion. There is considerable flexibility in the magnitude of the DM singlet fermion mass, which might itself might be generated by the interaction with the D-foam. It is suppressed hierarchically by the coupling $\alpha_{V}$ of the DM with the D-photon, whose value is poorly constrained, but may be enhanced hierarchically by geometrical effects in a multibraneworld scenario [37], as discussed in [16].

An important difference from the millicharged DM scenario is that both ionized and electrically-neutral matter particles are "charged" under the recoil D-matter gauge group $\mathrm{U}(1)_{D}$. Hence there is a coupling of DM singlet fermions with the bound electrons and protons of the hydrogen atoms that constitute most of the baryonic content of the Universe. The constraints on D-photon interactions are therefore very different from those on millicharged DM, avoiding most of the CMB constraints [21].

Detailed phenomenological studies are beyond the scope of this paper, but this exploratory study shows that $21-\mathrm{cm}$ astronomy might be relevant to the phenomenology of Dfoam. We recall that this is characterized by a D-particle density that is redshift-dependent, in general. D-particles can affect the refractive index of photons, which is constrained after recombination by measurements of the arrival times of high-energy photons from intense astrophysical sources such as gamma ray bursts, active galactic nuclei, along the lines suggested in previous works [9]. However, these constraints do not apply to the era before recombination that is accessible to $21-\mathrm{cm}$ astronomy.

We have made no claims here about the interpretation of the EDGES data [27], which is hotly debated [28]. Rather, our aim here has been to put some ideas on the table, suggesting scenarios in which (part of the) dark matter/dark energy in the Universe is of space-time foamy origin, which might have interesting phenomenological interfaces with future developments in $21-\mathrm{cm}$ astronomy.

\section{ACKNOWLEDGMENTS}

The work of J. E. and N. E. M. is supported in part by the U.K. Science and Technology Facilities Council (STFC) via the Grant No. ST/L000258/1, and that of J. E. is also supported in part by the Estonian Research Council via a Mobilitas Pluss grant. N.E. M. also acknowledges the current hospitality of IFIC Valencia through a Scientific Associateship (Doctor Vinculado). The work of D. V. N. is supported in part by DOE Grant No. DE-FG0213ER42020 and in part by the Alexander S. Onassis Public Benefit Foundation.

\section{APPENDIX: LENSING EFFECTS OF D-FOAM}

We comment in this Appendix on a complementary aspect of D-foam arising from its back reaction on the space-time geometry through which baryons and other matter particles propagate.

As discussed in [40], the recoil of the massive D-particle defects during their encounter with a propagating particle causes the space-time "seen" by the baryon at large scales to appear topologically nontrivial, deviating from Minkowski by a small solid-angular surplus: 


$$
\begin{aligned}
d s^{2} & =d t^{2}-d r^{2}-b^{2} r^{2}\left(\sin ^{2} \theta d \phi^{2}+d \theta^{2}\right), \\
b^{2} & =\frac{1}{1-\Delta^{2}}, \quad \Delta^{2} \ll 1,
\end{aligned}
$$

in standard spherical polar space-time coordinates, where

$$
\Delta^{2}=\left\langle|\vec{u}|^{2}\right\rangle_{\text {recoil }}=g_{s}^{2} \sigma^{2} \frac{|\overline{\vec{p}}|^{2}}{M_{s}^{2}}
$$

is the variance of the recoil velocity of the D-particle of mass $M_{s} / g_{s}$, the average $\langle\ldots\rangle$ is taken over the statistical ensemble of the D-particle defect populations, and $\overline{\vec{p}}$ denotes an average particle momentum.

Assuming a momentum transfer during an individual scattering $\Delta p_{i}=r_{i} p_{i}, r_{i}<1, i=1,2,3$ (no sum over $i$ ), then $\sigma^{2}$ denotes the stochastic variance for $r_{i}$, of the form $\left\langle r_{i} r_{j}\right\rangle=\sigma^{2} \delta_{i j},\left\langle r_{i}\right\rangle=0$. In the case of nonrelativistic thermalized baryons in a heat bath of temperature $T$, their average (thermal) momentum is $|\vec{p}|^{2} \sim 3 m_{b} T$, and (A2) leads to

$$
\Delta^{2}=\left\langle|\vec{u}|^{2}\right\rangle_{\text {recoil }} \sim \frac{3 \sigma^{2} m_{b} T}{M_{s}^{2}} g_{s}^{2},
$$

where $\sigma^{2}<1$ a phenomenological stochastic fluctuation parameter of the D-foam, that sets the scale for the "fuzziness" of space-time.

If one assumes that the ensemble of D-particles on our brane Universe are themselves thermalized at a temperature $T$, at least at late eras of the Universe [14], then one can define the following stochastic parameter related to their thermal velocity:

$$
\delta_{T}^{2}=\left\langle\left|\vec{u}^{(\mathrm{D})}\right|^{2}\right\rangle_{T},
$$

where $\vec{u}^{(\mathrm{D})}$ is the thermal velocity vector of a D-particle with mass $M_{s} / g_{s}$. The thermal velocity variance can be then estimated using the thermodynamic relation $\left\langle\frac{M_{s}}{g_{s}}\left|\vec{u}^{(D)}\right|^{2}\right\rangle_{T}=3 T$, yielding

$$
\delta_{T}^{2}=g_{s} \frac{3 T}{M_{s}}
$$

We note that this is much larger than the statistical fluctuations due to recoil momentum transfer under the same thermal conditions, shown in (A3), since $m_{b} \ll M_{s} / g_{s}$. For temperatures $T \sim 10 \mathrm{~K}$ typical of the cosmic dawn era and $M_{s} / g_{s}>10 \mathrm{TeV}$ (as implied by current collider limits from searches for extra dimensions) we have

$\delta_{T}^{2}<2.6 \times 10^{-16}, \quad \Delta^{2}<2.6 \times 10^{-20} \sigma^{2}, \quad \sigma^{2}<1$.
Thus there are at least four orders of magnitude difference between these two parameters of the D-foam. ${ }^{11}$

As discussed in [41,42], the propagation of particles in topologically nontrivial space-times of the form (A1) results in nontrivial scattering, whereby the defect "lenses" the particles, in the sense of inducing singular scattering amplitudes for scattering angles $\theta=\pi\left(1-b^{-1}\right)$. Such cross sections are not of Coulombic type, in the sense of scaling as $|\vec{p}|^{-2}$ rather than $|\vec{p}|^{-4}$, where $|\vec{p}|$ is the magnitude of the spatial momentum of the particle in the frame where the defect is at rest, in contrast to the standard millicharged DM interactions that scale like $v^{-4}$. As was carefully demonstrated in [42], these cross sections vanish in the no-defect limit so, for sufficiently small $\Delta^{2} \ll 1$, the corresponding differential cross sections are suppressed compared, for instance, with the cross sections discussed in the main text of this paper. Nonetheless, such an effect should in general be taken into account, especially in Type-IA models of D-foam, where there is no direct interaction of baryons with the D-particles. In such models the baryons simply feel the effects associated with the nontrivial topology of the "fuzzy" space time (A1) via the induced cross sections of [41,42]. In addition, in such space-times, the separation of the energy levels of atoms such as hydrogen, of relevance to us here, will be modified compared to the nondefect limit [43]. Thus, constraints on the strength of $\Delta^{2}$ could in principle be imposed in the framework of the $21-\mathrm{cm}$ astronomy. However, the parameter $\sigma$ is expected to be strongly suppressed, so indirect constraints on other parameters of the D-foam, as discussed in the main text, are expected to be much stronger.

We illustrate the above ideas by considering the scattering of a fermion in a space-time (A1), as studied by Ren in [41]. The scattering amplitude induced by a defect is given by

$$
\begin{aligned}
f(\theta) & =\frac{i}{|\vec{p}|} \delta(1-\cos \theta)+\frac{\sin (\pi \tilde{\alpha})(1-\cos \theta)^{1 / 2}}{2 \sqrt{2}|\vec{p}|(\cos (\pi \tilde{\alpha})-\cos \theta)^{3 / 2}}, \\
\tilde{\alpha} & \equiv 1-b^{-1},
\end{aligned}
$$

and the corresponding differential cross section is

$$
\frac{d \sigma}{d \Omega}=|f(\theta)|^{2}=\frac{\sin ^{2}(\pi \tilde{\alpha})(1-\cos \theta)}{8|\vec{p}|^{2}(\cos (\pi \tilde{\alpha})-\cos \theta)^{3}}+\ldots
$$

\footnotetext{
${ }^{11}$ The reader should notice that for relativistic particles, such as photons, for which the average thermal momentum scales as $|\vec{p}| \sim T$, there is a much stronger suppression of the parameter $\Delta^{2}$ compared to $\delta_{T}^{2}$, by at least sixteen orders of magnitude in the conditions considered here. Hence the slow-moving baryons provide much more sensitive probes of the foam parameter $\Delta^{2}$, and the topologically non-trivial structure of 'fuzzy' space-time (A1).
} 
where the ... represent singular terms arising from the $\delta$ function term in the scattering amplitude (A7), which need proper regularization in the $\theta=0$ (forward scattering) region, as discussed in [42]. This is essential for yielding the correct vanishing result for the scattering amplitude in the no-defect limit, where $\left.f(\theta)\right|_{\text {no-defect }}=0$, and hence the suppression of the cross sections for vanishingly small deficits. Notice also the singularity when $\theta=\pi \tilde{\alpha}$ of the second term in (A8), which is independent of the spin of the particle [41], and leads to the lensing effect on the particle.

The integration over the scattering angle $\theta$ encountered in the calculation of the total cross section exhibits singular behaviours in (A8) at the values of the scattering angle $\theta=\{0, \pi \tilde{\alpha}\}$ due to the first and second terms of (A8), respectively. For the small $\pi \tilde{\alpha} \ll 1$ that characterize our D-foam, one may impose a cutoff $\theta_{\min }$ for the $\theta$ angle to prevent it from approaching zero: $\theta_{\min }>\pi \tilde{\alpha} \neq 0$, which automatically regularizes the $\delta$-function term in (A8). However, this still leaves the other singular limit $\theta \rightarrow \pi \tilde{\alpha}$. In this case, we may regularize the denominator of the second term on the right-hand side of (A8) by means of an angular resolution cutoff $\Delta$, independent of $\pi \tilde{\alpha}$, such that

$$
\cos (\pi \tilde{\alpha})-\cos \theta \stackrel{\theta \rightarrow \pi \tilde{\alpha}}{\simeq} \mathcal{D}^{2} \ll 1
$$

In our foam case, we can approximate $\pi \tilde{\alpha} \simeq \frac{\pi}{2} \Delta^{2} \ll 1$, so that $\cos (\pi \tilde{\alpha}) \simeq 1-\frac{(\pi \tilde{\alpha})^{2}}{2}$. As we demonstrate below, this is a self-consistent approach that leads to finite and wellbehaved cross sections, which vanish in the no-defect limit, as discussed in [42].

The angular resolution $\mathcal{D}$ is assumed to be provided by the thermal motion of the D-foam, in the sense of the uncertainty in the scattering angle induced by the thermal fluctuations of the scatterer, which are considered much stronger than any inherent quantum-gravity effect. Hence, for our purposes, we can identify

$$
\mathcal{D}^{2}=\delta_{T}^{2}=g_{s} \frac{3 T}{M_{s}},
$$

with $\delta_{T}^{2}$ given in (A5), with

$$
1 \gg \mathcal{D} \gg \pi \tilde{\alpha}=\frac{\pi}{2} \Delta^{2}
$$

in view of (A6).

For the calculation of the corresponding transport cross section (3.1) of relevance to us here, it suffices to ignore the $\delta$-function term in (A8), which can be easily justified by assuming a minimum cutoff for the angular integration variable $\theta>\pi \tilde{\alpha})$. Concentrating on the second term, we may make a formal change of integration variable to $y=\cos (\pi \tilde{\alpha})-\cos \theta \simeq 1-\cos \theta-\frac{(\pi \tilde{\alpha})^{2}}{2}$, and write (by taking into account that $\theta \in(\pi \tilde{\alpha}, \pi])$

$$
\begin{aligned}
\sigma_{t} & =\int_{\frac{(\pi \tilde{\alpha})^{2}}{2}}^{-1} d(\cos \theta)(1-\cos \theta) \frac{d \sigma(\theta)}{d(\cos \theta)} \\
& \simeq \frac{(\pi \alpha)^{2}}{8|\vec{p}|^{2}} \int_{0}^{2} d y \frac{\left(y+\frac{(\pi \tilde{\alpha})^{2}}{2}\right)^{2}}{y^{3}},
\end{aligned}
$$

to leading order in $\pi \tilde{\alpha}$. As already mentioned, we observe that this leads to a formal singularity for the value $\theta=\pi \tilde{\alpha}$ due to the "lensing" effect, which requires additional regularization. According to our previous discussion, we now regularize the lower $y$-integration bound by replacing 0 with $\mathcal{D}^{2}$ (A10). We then easily obtain for the transport cross section (3.1):

$$
\begin{aligned}
\sigma_{t} & =\int d(\cos \theta)(1-\cos \theta) \frac{d \sigma(\theta)}{d(\cos \theta)} \\
& \stackrel{\Delta^{2} \ll 1}{\simeq} \frac{(\pi \alpha)^{2}}{8|\vec{p}|^{2}}\left[\ln \left(\frac{2}{\mathcal{D}^{2}}\right)+(\pi \alpha)^{2}\left(\frac{1}{\mathcal{D}^{2}}-\frac{1}{2}\right)+\frac{(\pi \alpha)^{4}}{8}\left(\frac{1}{\mathcal{D}^{4}}-\frac{1}{4}\right)\right] \\
& \simeq \frac{\pi^{2} \Delta^{4}}{32 m_{b}^{2}} \ln \left(7.6 \times 10^{15}\right) \frac{1}{v^{2}}+\ldots \\
& \simeq 1.07 \frac{\pi^{2} \Delta^{4}}{m_{b}^{2}} \frac{1}{v^{2}}+\ldots,
\end{aligned}
$$

where $\ldots$ denote subleading terms, $m_{b}$ is a generic baryon mass and $v$ is a relative velocity of the baryon with respect to the D-partlcle scatterer. Given that $\Delta^{2}$ is sufficiently small [cf. (A3), (A6)], we observe that this is suppressed compared to (3.3) as we mentioned previously, despite the $v^{-2}$ behavior.

We note for completeness that, with our regularization, the total cross section $\sigma_{\text {total }}=\int d \Omega|f(\theta)|^{2}$ is

$$
\begin{aligned}
\sigma_{\mathrm{tot}} & \simeq \frac{(\pi \alpha)^{2}}{8|\vec{p}|^{2}} \int_{0 \rightarrow D^{2}}^{2} d y \frac{y+\frac{(\pi \tilde{\alpha})^{2}}{2}}{y^{3}} \\
& \simeq \frac{(\pi \tilde{\alpha})^{2}}{8|\vec{p}|^{2}}\left[\frac{1}{\mathcal{D}^{2}}-\frac{1}{2}+\frac{(\pi \tilde{\alpha})^{2}}{4}\left(\frac{1}{\mathcal{D}^{4}}-\frac{1}{4}\right)\right],
\end{aligned}
$$

which is finite like the transport cross section and, as with $\sigma_{t}$, also vanishes in the no-defect limit $\tilde{\alpha} \rightarrow 0$, providing a self-consistency check of the approach [42].

In Type-I D-foam models, where the baryons do not interact directly with the foam, the effect (A13) is the dominant effect of the foam on baryonic matter. In such a case, one obtains a transport cross-section of the form $\sigma_{0} v^{-2}$, which may contribute to baryon cooling. From CMB limits, following the analysis of the second paper in [21], we know that with such a velocity dependence of the transport cross section between baryons and dark matter, one has $\sigma_{0} \lesssim 2.3 \times 10^{-33} \mathrm{~cm}^{2}$ at $95 \%$ C.L., implying on account of (A13) that

$$
\Delta^{2}<7.5 \times 10^{-4}, \quad \text { at } 95 \% \text { C.L. }
$$


As already mentioned, this constraint can be combined with the effect on hydrogen hyperfine splitting of the topologically nontrivial space-time (A1) following the analysis in [43], which will in general modify the features of the 21-cm emission spectrum. The limit (A15) is much weaker than the theoretically expected bound (A6). On the other hand, had we used the constraint on $\sigma_{0}$ imposed by CMB considerations [21] on Coulombic cross sections $\sigma_{0} v^{-4}$ between $\mathrm{DM}$ and baryons, $\sigma_{0}<1.7 \times 10^{-41} \mathrm{~cm}^{-2}$, we would have derived an experimental bound on $\Delta^{2}$ four orders of magnitude stronger than (A15), though still much weaker than the theoretical value (A6). It is for this reason that the consideration in the main text using the foam as a mediator between baryons and particle DM, lead to much stronger bounds on the relevant foam parameters, such as its density at a given redshift.

However, there are several steps that they need to be taken into account before the above ideas acquire a concrete shape and lead to a detailed phenomenology. These include a detailed study of the effects of D-foam with the above parameters on the CMB spectrum and the associated polarisations, given that the photons do interact with it, the effect of the drag on baryons on baryon acoustic oscillations, and additional astrophysical sources that may heat the baryons, counteracting their cooling and hence introducing uncertainties in the interpretation of the signal reported by EDGES [21], etc.
[1] G. Bertone and D. Hooper, Rev. Mod. Phys. 90, 045002 (2018).

[2] B. P. Abbott et al. (LIGO Scientific and Virgo Collaborations), Phys. Rev. Lett. 116, 061102 (2016).

[3] M. Milgrom, Astrophys. J. 270, 365 (1983).

[4] J. A. Wheeler, Phys. Rev. 97, 511 (1955).

[5] J. Polchinski, String Theory. Vol. 1: An Introduction to the Bosonic String (Cambridge University Press, Cambridge, England, 1998); String Theory. Vol. 2: Superstring Theory and Beyond (Cambridge University Press, Cambridge, England, 1998).

[6] J. R. Ellis, N. E. Mavromatos, and D. V. Nanopoulos, Gen. Relativ. Gravit. 32, 127 (2000); Phys. Rev. D 61, 027503 (1999); 62, 084019 (2000).

[7] J. R. Ellis, N. E. Mavromatos, and M. Westmuckett, Phys. Rev. D 70, 044036 (2004); 71, 106006 (2005); J. R. Ellis, N. E. Mavromatos, D. V. Nanopoulos, and M. Westmuckett, Int. J. Mod. Phys. A 21, 1379 (2006).

[8] J. R. Ellis, N. E. Mavromatos, and A. S. Sakharov, Astropart. Phys. 20, 669 (2004); J. R. Ellis, N. E. Mavromatos, D. V. Nanopoulos, and A. S. Sakharov, Nature (London) 428, 386 (2004); Int. J. Mod. Phys. A 19, 4413 (2004).

[9] J. R. Ellis, N. E. Mavromatos, and D. V. Nanopoulos, Phys. Lett. B 665, 412 (2008); Int. J. Mod. Phys. A 26, 2243 (2011); Phys. Lett. B 694, 61 (2010).

[10] T. Li, N. E. Mavromatos, D. V. Nanopoulos, and D. Xie, Phys. Lett. B 679, 407 (2009).

[11] N. E. Mavromatos, Phys. Rev. D 83, 025018 (2011).

[12] J. Polchinski, arXiv:hep-th/9611050.

[13] N. E. Mavromatos, S. Sarkar, and A. Vergou, Phys. Lett. B 696, 300 (2011); N.E. Mavromatos, V. A. Mitsou, S. Sarkar, and A. Vergou, Eur. Phys. J. C 72, 1956 (2012).

[14] N.E. Mavromatos, M. Sakellariadou, and M. F. Yusaf, J. Cosmol. Astropart. Phys. 03 (2013) 015; T. Elghozi, N. E. Mavromatos, M. Sakellariadou, and M. F. Yusaf, J. Cosmol. Astropart. Phys. 02 (2016) 060.

[15] I. I. Kogan, N.E. Mavromatos, and J. F. Wheater, Phys. Lett. B 387, 483 (1996); J. R. Ellis, N. E. Mavromatos, and
D. V. Nanopoulos, Int. J. Mod. Phys. A 13, 1059 (1998); N. E. Mavromatos and R. J. Szabo, Phys. Rev. D 59, 104018 (1999).

[16] J. Ellis, N. E. Mavromatos, and D. V. Nanopoulos, Phys. Rev. D 96, 086012 (2017).

[17] G. Amelino-Camelia, J. R. Ellis, N. E. Mavromatos, and D. V. Nanopoulos, Int. J. Mod. Phys. A 12, 607 (1997); G. Amelino-Camelia, J. R. Ellis, N. E. Mavromatos, D. V. Nanopoulos, and S. Sarkar, Nature (London) 393, 763 (1998).

[18] For a review on this topic, see N. E. Mavromatos, Int. J. Mod. Phys. A 25, 5409 (2010), and references therein.

[19] R. Barkana, Nature (London) 555, 71 (2018); A. Fialkov, R. Barkana, and A. Cohen, Phys. Rev. Lett. 121, 011101 (2018); P. Sharma, Mon. Not. R. Astron. Soc. 481, L6 (2018).

[20] J. B. Muñoz and A. Loeb, Nature (London) 557, 684 (2018).

[21] E. D. Kovetz, V. Poulin, V. Gluscevic, K. K. Boddy, R. Barkana, and M. Kamionkowski, Phys. Rev. D 98, 103529 (2018); K. K. Boddy, V. Gluscevic, V. Poulin, E. D. Kovetz, M. Kamionkowski, and R. Barkana, Phys. Rev. D 98, 123506 (2018).

[22] S. D. McDermott, H. B. Yu, and K. M. Zurek, Phys. Rev. D 83, 063509 (2011); C. Dvorkin, K. Blum, and M. Kamionkowski, Phys. Rev. D 89, 023519 (2014).

[23] J. B. Muñoz, E. D. Kovetz, and Y. Ali-Haïmoud, Phys. Rev. D 92, 083528 (2015).

[24] L. B. Jia, arXiv:1804.07934.

[25] N. Houston, C. Li, T. Li, Q. Yang, and X. Zhang, Phys. Rev. Lett. 121, 111301 (2018).

[26] P. Sikivie, arXiv:1805.05577.

[27] J. D. Bowman, A. E. E. Rogers, R. A. Monsalve, T. J. Mozdzen, and N. Mahesh, Nature (London) 555, 67 (2018).

[28] R. Hills, G. Kulkarni, P. D. Meerburg, and E. Puchwein, arXiv: 1805.01421.

[29] L. B. Okun, Sov. Phys. JETP 56, 502 (1982) [Zh. Eksp. Teor. Fiz. 83, 892 (1982)]; P. Galison and A. Manohar, Phys. Lett. 136B, 279 (1984); P. Fayet, Nucl. Phys. B347, 743 
(1990); R. Foot and S. Vagnozzi, Phys. Lett. B 748, 61 (2015); S. Bilmis, I. Turan, T. M. Aliev, M. Deniz, L. Singh, and H. T. Wong, Phys. Rev. D 92, 033009 (2015); J. L. Feng, J. Smolinsky, and P. Tanedo, Phys. Rev. D 93, 015014 (2016); 96, 099901(E) (2017).

[30] O. Erken, P. Sikivie, H. Tam, and Q. Yang, Phys. Rev. D 85, 063520 (2012); P. Sikivie and Q. Yang, Phys. Rev. Lett. 103, 111301 (2009).

[31] A. Arvanitaki, S. Dimopoulos, S. Dubovsky, N. Kaloper, and J. March-Russell, Phys. Rev. D 81, 123530 (2010); M. Cicoli, M. Goodsell, and A. Ringwald, J. High Energy Phys. 10 (2012) 146.

[32] C. R. Argüelles, N. E. Mavromatos, J. A. Rueda, and R. Ruffini, J. Cosmol. Astropart. Phys. 04 (2016) 038; N. E. Mavromatos, C. R. Argüelles, R. Ruffini, and J. A. Rueda, Int. J. Mod. Phys. D 26, 1730007 (2017).

[33] B. Moore, Nature (London) 370, 629 (1994); W. J. G. de Blok, Adv. Astron. 2010, 789293 (2010); A. A. Klypin, A. V. Kravtsov, O. Valenzuela, and F. Prada, Astrophys. J. 522, 82 (1999); E. Polisensky and M. Ricotti, Phys. Rev. D 83, 043506 (2011); B. Moore, S. Ghigna, F. Governato, G. Lake, T. R. Quinn, J. Stadel, and P. Tozzi, Astrophys. J. 524, L19 (1999); M. Boylan-Kolchin, J. S. Bullock, and M. Kaplinghat, Mon. Not. R. Astron. Soc. 415, L40 (2011); M. Boylan-Kolchin, J. S. Bullock, and M. Kaplinghat, Mon. Not. R. Astron. Soc. 422, 1203 (2012).
[34] G. Shiu and L. T. Wang, Phys. Rev. D 69, 126007 (2004).

[35] L. Andrianopoli, R. D'Auria, and M. Trigiante, Phys. Lett. B 744, 225 (2015); See also: S. Ferrara, A. Sagnotti, and A. Yeranyan, J. High Energy Phys. 05 (2015) 051; Nucl. Phys. B912, 305 (2016).

[36] See, for instance: G. Arcadi, Y. Mambrini, and F. Richard, J. Cosmol. Astropart. Phys. 03 (2015) 018, and references therein.

[37] N. E. Mavromatos and J. Rizos, Int. J. Mod. Phys. A 18, 57 (2003); Phys. Rev. D 62, 124004 (2000).

[38] L. Randall and R. Sundrum, Phys. Rev. Lett. 83, 3370 (1999).

[39] P. A. R. Ade et al. (Planck Collaboration), Astron. Astrophys. 594, A13 (2016).

[40] N. E. Mavromatos, Found. Phys. 40, 917 (2010).

[41] P. O. Mazur and J. Papavassiliou, Phys. Rev. D 44, 1317 (1991); The discussion in that work was mainly for scalar neutral particles. Extensions to include other spins and charge effects have been considered in: H. Ren, Phys. Lett. B 325, 149 (1994); E. R. B. de Mello and C. Furtado, Phys. Rev. D 56, 1345 (1997); A. A. Roderigues Sobreira and E. R. Bezerra de Mello, Gravitation Cosmol. 5, 177 (1999).

[42] N. E. Mavromatos and J. Papavassiliou, Eur. Phys. J. C 78, 68 (2018).

[43] G. A. Marques and V. B. Bezerra, Phys. Rev. D 66, 105011 (2002). 